\title{
Revisión de tema
}

\section{Manifestaciones dermatológicas de COVID-19: ¿casualidad o causalidad?}

\author{
Cutaneous manifestations of COVID-19: ¿chance or causality?
}

Maria Isabel Arredondo ${ }^{1 \otimes}$, Lina Vanesa Gomez ${ }^{2 \text { cvLAC }}$, Delsy Yurledy Del Rio C ${ }^{3}$

Fecha correspondencia:

Recibido: mayo 12 de 2020.

Revisado: junio 24 de 2020.

Aceptado: julio 1 de 2020.

Forma de citar:

Arredondo Ml, Gómez lv, Del Rio DY. Manifestaciones dermatológicas de COVID-19: ¿casualidad o causalidad? Rev CES Med. 2020; Especial COVID-19: 34-41.

\section{Open access}

(C) Derecho de autor

Licencia creative commons

Ética de publicaciones

Revisión por pares

Gestión por Open Journal System

DOl: http://dx.doi.org/10.21615/

cesmedicina.34.COVID-19.7

ISSN 0120-8705

e-ISSN 2215-9177

Sobre los autores:

1. Dermatóloga. Hospital Pablo Tobón Uribe, Medellín, Colombia.

2. Dermatóloga Hospital Pablo Tobón Uribe, Medellín, Colombia.

Comparte

\section{Resumen}

En esta revisión narrativa de la literatura se describen las manifestaciones en piel del SARS-CoV-2 en todas las edades reportadas hasta hoy. El objetivo es entender la presentación clínica y el tiempo de aparición en el curso de la enfermedad, para aumentar el rendimiento de las pruebas diagnósticas y mejorar la identificación de pacientes asintomáticos. Para la descripción se clasifican en cinco grupos: relacionadas directa o indirectamente con el virus, toxicodermias, dermatitis de contacto y trauma por presión. Hasta ahora no se ha comprobado si estas lesiones son por una manifestación directa del virus, por una reacción inmunitaria inespecífica o secundarias a los tratamientos usados.

Palabras clave: Infecciones por coronavirus; Enfermedades de la piel; Toxicodermias.

\begin{abstract}
This narrative review describes the manifestations of SARS-CoV-2 in skin at all ages reported to date. The objective is to understand the clinical presentation and time of onset in the course of the disease, to increase the performance of diagnostic tests and improve the identification of asymptomatic patients. They are classified into five groups: directly or indirectly related to the virus, toxicoderma, contact dermatitis and pressure trauma. Until now it has not been verified whether these lesions are due to a direct manifestation of the virus or due to an immune, nonspecific reaction or the treatments used.
\end{abstract}

Keywords: Coronavirus infections; Skin diseases; Toxicoderma.

\section{Introducción}

En diciembre de 2019 apareció en Wuhan, China, una neumonía epidémica causada por un nuevo beta coronavirus, el SARS-CoV-2. El 11 de marzo de 2020 la Organización Mundial de la Salud (OMS) lo declaró una pandemia. El objetivo de esta revisión de la literatura es describir las manifestaciones dermatológicas del virus reportadas hasta hoy, se postula que puedan ser útiles para identificar posibles pacientes, realizar un diagnóstico temprano y evitar la trasmisión y diseminación de la enfermedad. 
3. Dermatóloga Universidad de Antioquia. Hospital Pablo Tobón Uribe, Medellín, Colombia.
Es importante ser cuidadosos en la interpretación de lesiones dermatológicas asociadas a COVID-19 y evaluar si realmente son manifestaciones específicas del virus o son secundarias a coinfección con otros virus o por toxicodermias (2).

\section{Metodología}

Esta revisión se realizó a partir de artículos disponibles en bases de datos en línea como Embase y PubMed. Se incluyeron documentos publicados hasta mayo de 2020. Se tomaron en cuenta cartas al editor, reportes de caso, series de caso y artículos originales en inglés y español con las palabras piel, SARS-CoV-2 y COVID-19.

\section{Manifestaciones en piel}

La primera descripción de lesiones cutáneas por SARS-CoV-2 es realizada en Italia por Recalcati, et al, quienes reportan 88 pacientes, de los cuales 18 (20,4\%) tenían lesiones en piel así: rash eritematoso 14 pacientes, urticaria tres pacientes y vesículas similares a la varicela un paciente (1).

Es importante ser cuidadosos en la interpretación de lesiones dermatológicas asociadas a COVID-19 y evaluar si realmente son manifestaciones específicas del virus o son secundarias a coinfección con otros virus o por toxicodermias (2).

Las condiciones dermatológicas que se relacionan con COVID-19 se enumerarán en cinco grupos (I-V). Las dos primeras causadas "probablemente" por SARS-CoV-2 y relevantes para el diagnóstico. Los otros grupos se desarrollan secundariamente.

\section{I) Relacionadas indirectamente con el SARS-COV-2}

Se postula que el SARS-CoV-2 induce una respuesta inflamatoria sistémica con secreción masiva de citoquinas proinflamatorias que llevan a una vasculitis de medianos y pequeños vasos. La clínica no es característica y simula otras infecciones virales. Las lesiones pueden aparecer antes o después de los síntomas sistémicos y tienen distintas manifestaciones $(3,4)$.

Rash o exantema maculopapular eritematoso: es inespecífico, puede presentarse como rash típico con máculas purpúricas eritematosas confluentes, pero en ocasiones se manifiesta con lesiones purúrpicas, simulando otras dermatosis pruriginosas o no (5).

También puede comprometer zonas flexurales, similares al exantema intertriginoso y flexural simétrico, secundario a medicamentos o a virus como el parvovirus B19 $(6,7)$. Además se han descrito casos de rash petequial similar al dengue (8) y en un caso, lesiones anulares en cara y tronco superior asociadas a fiebre (9).

En la mayoría de los artículos no han sido descritos los hallazgos histopatológicos. Gianotti, et al. realizaron biopsias a tres pacientes positivos para COVID-19 con lesiones en piel y observaron en dos de ellos hallazgos similares a los vistos en otros exantemas virales, con dermatitis perivascular en la dermis superficial o profunda. En el primer paciente asociado a vasos trombosados, edematosos, con neutrófilos, eosinófilos y polvo nuclear dérmico. En el segundo, se evidenciaron cúmulos de linfocitos perivasculares en patrón vasculítico y eritrocitos extravasados; y en el tercero hendiduras acantolíticas focales suprabasales, disqueratosis y signos de balonización de las células simulando una infección herpética, asociados a una dermatitis de interfase con algunos queratinocitos necróticos rodeados por linfocitos que sugieren que el virus produce activación en la cascada de citoquinas y de linfocitos citotóxicos natural killer (10).

Hay otro artículo que reporta hallazgos histopatológicos similares asociados a vasos dilatados en dermis papilar y media (11). 
Las manifestaciones cutaneas relacionadas directamente con SARS-CoV-2 son producidas por la interacción viral con células endoteliales, causando microtrombos.
Se propone que este exantema ocurre por diseminación hematógena viral por el sistema vascular cutáneo, activación del sistema inmune y movilización de linfocitos y células de Langerhans, creación de complejos inmunes y daño endotelial (12).

Otras enfermedades virales como el sarampión, rubeola y dengue causan rash en la piel, con un patrón de presentación definido en el momento de aparición respecto a la viremia. En la infección por el SARS CoV2 esto aún es desconocido (13).

Urticaria: descrita en pacientes sintomáticos y asintomáticos. Se presenta con habones pruriginosos en la cara, zonas acrales y extremidades. En tres de los casos los habones estuvieron presentes antes de los síntomas sistémicos y se acompañaron de pirexia (14).

Pápulas y placas eritemato amarillosas en sitios de presión: encontradas en una paciente en los tobillos después del diagnóstico de COVID 19 (15).

Pitiriasis rosada: descrita en Irán en un paciente con lesiones compatibles con pitiriasis rosada y antecedente de contacto con SARS-CoV-2 (16).

Erupción variceliforme: se presenta con lesiones tipo vesículas y menos frecuentemente pápulas eritematosas que progresan a vesículas rodeadas por un halo eritematoso, muy poco pruriginosas, predominantemente en el tronco y menos en extremidades, sin afectar la cara ni mucosas y con una duración promedio de ocho días. Observadas antes y después del inicio de los síntomas sistémicos (17). No hay claridad en estos reportes de pruebas para excluir infección herpética.

\section{II) Relacionadas directamente con el SARS- CoV-2}

Producidas por la interacción viral con células endoteliales, causando microtrombos, vasculopatía y daño endotelial. Se sugiere que un estado hipercoagulable explicaría las lesiones de piel y en otros órganos. Estudios de patología de piel y pulmones han encontrado depósitos de complemento C5b-9 y C4d que generan una enfermedad microtrombótica inflamatoria mediada por complemento (3). Se manifiesta con cianosis periférica por el estado protrombótico, palidez distal, pápulas y placas eritemato violáceas, ampollas, necrosis y ulceración.

Livedo reticularis transitoria: reportada en dos pacientes con COVID-19 en Estados Unidos. El primero, de 67 años, luego de una semana de síntomas respiratorios, presentó un parche liveloide unilateral traslúcido no pruriginoso, en la cara anterolateral de muslo derecho que desapareció espontáneamente en 19 horas. La segunda, de 47 años, 10 días después de una prueba positiva de COVID-19, presentó rash livedoide asintomático en su pierna derecha, con una duración de 20 minutos y autoresolución. El livedo reticularis es causado por condiciones como la coagulación intravascular diseminada que reduce el flujo sanguíneo microvascular cutáneo, con acumulación de sangre desoxigenada evidenciada con lesiones violáceas y transitorias (18).

Lesiones isquémicas y acroisquémicas: reportadas en adultos de unidades de cuidado intensivo en Wuhan, con lesiones eritemato - violáceas azuladas isquémicas y cianosis en pies, manos o plantas, algunos con ampollas hemorrágicas o costras necróticas (19); luego descritas en dos adultos de Catar con lesiones purpúricas subungueales (20). 
Se postula que el coronavirus induce un síndrome hiperinflamatorio similar a la enfermedad de Kawasaki.
En Italia describieron 63 casos con una media de edad de 14 años, con lesiones eritematoedematosas, ampollosas, asociadas a dolor o prurito localizadas en pies en el $85 \%$, manos y pies en el $7 \%$ y manos en el $6 \%$. No todos los pacientes tuvieron confirmación diagnóstica de COVID 19 (21).

Luego, también en Italia, reportan lesiones acroisquémicas de características similares en cuatro niños (22) y Recalcati et al. reportan 14 casos, 11 de ellos niños con lesiones acrales maculopapulares eritemato - violáceas en pies y manos, y lesiones en diana en manos y codos, con autoresolución en dos a cuatro semanas. Las biopsias de cuatro de ellos reportaron infiltrado linfocítico denso en dermis superficial e hipodermis de predominio perivascular y signos de activación endotelial. Se descartaron otras causas virales, pero la PCR para SARS-CoV-2 19 fue negativa (23).

En Francia reportaron lesiones maculares, violáceas, en porcelana, lesiones purpúricas necróticas y no necróticas, angiomas, fenómeno de Raynoud y lesiones tipo sabañones (chillblains like) en siete de 14 pacientes observados (24).

En España, un estudio retrospectivo de 132 pacientes con contacto cercano con confirmados de COVID-19, describe dos patrones de lesiones acroisquémicas que pueden superponerse: el primero es un patrón similar al chilblain (sabañones) con máculas rojas a violáceas, placas y nódulos, generalmente localizados en los dedos de manos y pies. El segundo, similar al eritema multiforme con máculas eritematosas redondeadas y vesículas que tienden a fusionarse. Comparado con el eritema multiforme clásico, las lesiones individuales son más pequeñas $(<1 \mathrm{~cm})$, menos extensas y sin las lesiones en diana típicas. Solo dos pacientes presentaron también lesiones en otros sitios como codos, rodillas y orejas (25).

Aunque en muchos de los casos con lesiones acroisquémicas no se ha confirmado la infección por SARS-CoV-2, el soporte diagnóstico se realizó por el nexo epidemiológico y la resolución espontánea de los síntomas. Estas lesiones no son frecuentes en otras enfermedades como la perniosis, la vasculitis de Henoch-Schoenlein, la sepsis meningocócica o la deficiencia de proteína $\mathrm{C}$, lo que hace que este sea un signo dermatológico útil en identificar a los niños y adolescentes con formas mínimas de infección (26).

Respecto a los hallazgos histopatológicos, se ha descrito en un paciente con lesiones de chilblain (sabañones) un infiltrado liquenoide perivascular y periecrino superficial y profundo. No se identificaron trombos de fibrina intraluminales en las vénulas. La inmunofluorescencia directa fue negativa (27).

Enfermedad de Kawasaki-like: se postula que el coronavirus induce un síndrome hiperinflamatorio similar a la enfermedad de Kawasaki. El primer reporte es de una niña de seis meses con PCR positiva para SARS-CoV-2, conjuntivitis, rash, edema en extremidades y fiebre persistente; tratada con inmunoglobulina intravenosa y ácido acetil salicílico con mejoría (28). Desde entonces se han descrito múltiples casos con criterios parciales o totales para enfermedad de Kawasaki; entre ellos, dos con fiebre persistente, diarrea, conjuntivitis, labios fisurados, rash, eritema, edema de manos y pies, elevación de marcadores inflamatorios, linfopenia, trombocitopenia y consumo de complemento; ambos con hisopado nasal para SARS-CoV-2 negativo y títulos altos de lgM e lgG; lo que sugiere una respuesta inflamatoria desarrollada en la fase más tardía de la enfermedad cuando el virus ya no es detectado en el tracto respiratorio superior. 
A la fecha no se ha comprobado si estas lesiones son una manifestación directa del virus, una reacción inmunitaria inespecífica o son secundarias a los tratamientos usados. Todas deben ser tenidas en cuenta para la posible identificación de pacientes de manera temprana y evitar que se trasmita la enfermedad. Se requieren más estudios para determinar y su especificidad para la enfermedad.
Estos casos cumplían criterios completos para enfermedad de Kawasaki, pero características inusuales como la edad de inicio y la trombocitopenia, hallazgos que son inusuales en la enfermedad de Kawasaki clásica, excepto cuando se desarrolla un síndrome de activación de macrófagos. Además, presentaron otras características diferenciales como diarrea prodrómica, síndrome capillary leak (choque, hemoconcentración, hipoalbuminemia) y disfunción miocárdica, sin compromiso coronario (33). Se cree que el compromiso mucocutáneo, el consumo del complemento y plaquetario, son ocasionados por la microvasculopatía inducida por el virus, que lleva a fuga capilar y una respuesta humoral retardada. Se propone seguimiento con ecocardiograma para evaluar aneurismas coronarios $(28,29,30)$.

\section{III) Toxicodermias}

El tratamiento para SARS-CoV2 puede disparar toxicodermias como vasculitis por uso de antibióticos, reacción medicamentosa con eosinofilia y síntomas sistémicos, y pustulosis exantemática generalizada, estas dos últimas descritas por uso de hidroxicloroquina $(31,32,33)$. No siempre es posible establecer si las manifestaciones cutáneas realmente corresponden a compromiso por COVID-19 o a una toxicodermia.

\section{IV) Dermatitis de contacto y V) trauma por presión}

Se presentan en otro artículo de esta revista (Compromiso cutáneo secundario a equipo del protección contra COVID19 en el personal de la salud).

\section{Conclusión}

Se reportan las manifestaciones del SARS CoV2 en piel publicadas hasta mayo de 2020. A la fecha no se ha comprobado si estas lesiones son una manifestación directa del virus, una reacción inmunitaria inespecífica o son secundarias a los tratamientos usados. Todas deben ser tenidas en cuenta para la posible identificación de pacientes de manera temprana y evitar que se trasmita la enfermedad. Se requieren más estudios para determinar y su especificidad para la enfermedad. De esta forma se podría determinar el momento óptimo para la toma de pruebas diagnósticas y aumentar su rendimiento.

\section{Conflictos de interés}

No se reportan conflictos de interés.

\section{Bibliografía}

1. Recalcati S. Cutaneous manifestations in COVID-19: a first perspective. J Eur Acad Dermatol Venereol [Internet]. 2020 [citado 2020 Abr 29]. Disponible en https://doi.org/10.1111/jdv.16387. [Epub ahead of print]

2. Madigan LM, Micheletti RG, Shinkai K. How Dermatologists Can Learn and Contribute at the Leading Edge of the COVID-19 Global Pandemic. JAMA Dermatol [Internet]. 2020 [citado 2020 Abr 29. Disponible en: https://doi.org/10.1001/jamadermatol.2020.1438. [Epub ahead of print]

3. Magro C, Mulvey JJ, Berlin D, Nuovo G, Salvatore S, Harp J, et al. Complement associated microvascular injury and thrombosis in the pathogenesis of severe COVID-19 infection: a report of five cases. Transl Res [Internet]. 2020 [citado 2020 Abr 29];S1931524420300700. Disponible en: https://doi.org/10.1016/j. trsl.2020.04.007. [Epub ahead of print] 
4. Galván Casas, C., Català, A., Carretero Hernández, G., Rodríguez-Jiménez, P., Fernández Nieto, D., Rodríguez-Villa Lario, et al. Classification of the cutaneous manifestations of COVID-19: a rapid prospective nationwide consensus study in Spain with 375 cases. Br J Dermatol [Internet]. 2020 [citado 2020 Abr 29]. Disponible en : https://doi.org/10.1111/bjd.19163. [Epub ahead of print]

5. Hedou M, Carsuzaa F, Chary E, Hainaut E, Cazenave-Roblot F, Masson Regnault M. Comment on "Cutaneous manifestations in COVID-19: a first perspective " by Recalcati S. J Eur Acad Dermatol Venereol [Internet]. 2020 [citado 2020 Abr 30]. Disponible en: https://doi.org/10.1111/jdv.16519. [Epub ahead of print]

6. Mahé A, Birckel E, Krieger S, Merklen C, Bottlaender L. A distinctive skin rash associated with Coronavirus Disease 2019?. J Eur Acad Dermatol Venereol [Internet]. 2020 [citado 2020 May 1]. Disponible en: https://doi.org/10.1111/jdv.16471. [Epub ahead of print]

7. Jimenez-Cauhe J, Ortega-Quijano D, Prieto-Barrios M, Moreno-Arrones OM, Fernandez-Nieto D. Reply to "COVID-19 can present with a rash and be mistaken for dengue": Petechial rash in a patient with COVID-19 infection. J Am Acad Dermatol [Internet]. 2020 [citado 2020 Abr 30];S0190962220305569. Disponible en: https://doi.org/10.1016/j.jaad.2020.04.016. [Epub ahead of print]

8. Joob B, Wiwanitkit V. COVID-19 can present with a rash and be mistaken for dengue. J Am Acad Dermatol [Internet]. 2020 [citado 2020 May 2];82(5):e177. Disponible en: https://doi.org/10.1016/j.jaad.2020.03.036

9. Amatore F, Macagno N, Mailhe M, Demarez B, Gaudy-Marqueste C, Grob J, et al. SARS-CoV-2 infection presenting as a febrile rash. J Eur Acad Dermatol Venereol [Internet]. 2020 [citado 2020 May 2];16528. Disponible en: https://doi. org/10.1111/jdv.16528. [Epub ahead of print]

10. Gianotti R, Zerbi P, Dodiuk-Gad RP. Clinical and histopathological study of skin dermatoses in patients affected by COVID-19 infection in the Northern part of Italy. J Dermatol Sci [Internet]. 2020 [citado 2020 May 3];S0923181120301432 Disponible en: https://doi.org/10.1016/i.jdermsci.2020.04.007. [Epub ahead of print]

11. Zengarini C, Orioni G, Cascavilla A, Horna Solera C, Fulgaro C, Misciali C, et al. Histological pattern in Covid-19 induced viral rash. J Eur Acad Dermatol Venereol [Internet]. 2020 [citado 2020 May 4]. Disponible en: https://doi.org/10.1111/ jdv.16569. [Epub ahead of print]

12. Ahouach B1, Harant S2, Ullmer A3, Martres P4, Bégon E1, Blum L1, Tess 05, Bachmeyer C6. Cutaneous lesions in a patient with COVID-19 are they related. Br J Dermatol [Internet]. 2020 [citado 2020 May 4]; 19168. Disponible en: https://doi. org/10.1111/bjd.19168. [Epub ahead of print]

13. Su C-J, Lee C-H. Viral exanthem in COVID-19, a clinical enigma with biological significance. J Eur Acad Dermatol Venereol [Internet]. 2020 [citado 2020 May 6]. Disponible en : https://doi.org/10.1111/jdv.16469. [Epub ahead of print] 
14. Van Damme C, Berlingin E, Saussez S, Accaputo O. Acute urticaria with pyrexia as the first manifestations of a COVID-19 infection. J Eur Acad Dermatol Venereol [Internet]. 2020 [citado 2020 May 7]. Disponible en: https://doi.org/10.1111/ jdv.16523. [Epub ahead of print]

15. Estébanez A, Pérez-Santiago L, Silva E, Guillen-Climent S, García-Vázquez A, Ramón MD. Cutaneous manifestations in COVID-19: a new contribution. J Eur Acad Dermatol Venereol [Internet]. 2020 [citado 2020 May 8]. Disponible en: https:// doi.org/10.1111/jdv.16474. [Epub ahead of print]

16. Ehsani AH, Nasimi M, Bigdelo Z. Pityriasis rosea as a cutaneous manifestation of COVID-19 infection. J Eur Acad Dermatol Venereol [Internet]. 2020 [citado 2020 May 8]. Disponible en: https://doi.org/10.1111/jdv.16579. [Epub ahead of print]

17. Genovese G, Colonna C, Marzano AV. Varicella-like exanthem associated with COVID-19 in an 8-year-old girl: A diagnostic clue? Pediatr Dermatol [Internet]. 2020 [citado 2020 May 8]. Disponible en: https://doi.org/10.1111/pde.14201. [Epub ahead of print]

18. Manalo IF, Smith MK, Cheeley J, Jacobs R. A dermatologic manifestation of COVID-19: Transient livedo reticularis. J Am Acad Dermatol [Internet]. 2020 [citado May 10]. Disponible en: https://doi.org/10.1016/j.jaad.2020.04.018. [Epub ahead of print]

19. LiT,LuH,ZhangW.ClinicalobservationandmanagementofCOVID-19 patients. Emerg Microbes Infect. 2020 Dec;9(1):687-690. doi: 10.1080/22221751.2020.1741327.

20. Alramthan A, Aldaraji W. A case of COVID-19 presenting in clinical picture resembling chilblains disease. First report from the Middle East. Clin Exp Dermatol [Internet]. 2020 [citado 2020 May 10]. Disponible en: https://doi.org/10.1111/ ced.14243. [Epub ahead of print]

21. Piccolo V, Neri I, Filippeschi C, Oranges T, Argenziano G, Battarra VC, et al. Chilblain-like lesions during COVID-19 epidemic: a preliminary study on 63 patients. J Eur Acad Dermatol Venereol [Internet]. 2020 [citado 2020 May 10]. Disponible en: https://doi.org/10.1111/jdv.16526. [Epub ahead of print]

22. Colonna C, Monzani Na, Rocchi A, Gianotti R, Boggio F, Gelmetti C. Chilblains-like lesions in children following suspected Covid-19 infection. Pediatr Dermatol [Internet]. 2020 [citado 2020 May 11]. Disponible en: https://doi.org/10.1111/ pde.14210. [Epub ahead of print]

23. Recalcati S, Barbagallo T, Frasin LA, Prestinari F, Cogliardi A, Provero MC, et al. Acral cutaneous lesions in the Time of COVID-19. J Eur Acad Dermatol Venereol [Internet]. 2020 [citado 2020 May 11]. Disponible en: https://doi.org/10.1111/ jdv.16533. [Epub ahead of print]

24. Bouaziz J, Duong T, Jachiet M, Velter C, Lestang P, Cassius C, et al. Vascular skin symptoms in COVID-19: a french observational study. J Eur Acad Dermatol Venereol [Internet]. 2020 [citado 2020 May 11] Disponible en: https://doi.org/10.1111/ jdv.16544. [Epub ahead of print] 
25. Fernandez-Nieto D, Jimenez-Cauhe J, Suarez-Valle A, Moreno-Arrones OM, Saceda-Corralo D, Arana-Raja A, et al. Characterization of acute acro-ischemic lesions in non-hospitalized patients: a case series of 132 patients during the COVID-19 outbreak. J Am Acad Dermatol [Internet]. 2020 [citado 2020 May 11]; S01909622(20)30709-X. Disponible en: https://doi.org/10.1016/j.jaad.2020.04.093. [Epub ahead of print]

26. López-Robles J, de la Hera I, Pardo J, Martínez J, Cutillas-Marco E. Chilblain-like lesions: a case series of 41 patients during the COVID-19 pandemic. Clin Exp Dermatol [Internet]. 2020 [citado 2020 May 12]. Disponible en: https://doi. org/10.1111/ced.14275. [Epub ahead of print]

27. Kolivras A, Dehavay F, Delplace D, Feoli F, Meiers I, Milone L, et al. Coronavirus (COVID-19) infection-induced chilblains: A case report with histopathologic findings. JAAD Case Rep [Internet]. 2020 [citado 2020 May 12]. Disponible en: https://doi. org/10.1016/j.jdcr.2020.04.011. [Epub ahead of print]

28. Jones VG, Mills M, Suarez D, Hogan CA, Yeh D, Segal JB, et al. COVID-19 and Kawasaki Disease: Novel Virus and Novel Case. Hosp Pediatr [Internet]. 2020 [citado 2020 May 27]; hpeds.2020-0123. Disponible en: https://doi.org/10.1542/ hpeds.2020-0123. [Epub ahead of print]

29. Licciardi F, Pruccoli G, Denina M, Parodi E, Taglietto M, Rosati S, et al. SARS-CoV-2Induced Kawasaki-Like Hyperinflammatory Syndrome: A Novel COVID Phenotype in Children. Pediatrics [Internet]. 2020 [citado 2020 May 27];:20201711. Disponible en: https://doi.org/10.1542/peds.2020-1711. [Epub ahead of print]

30. Schroeder AR, Wilson KM, Ralston SL. COVID-19 and Kawasaki Disease: Finding the Signal in the Noise. Hosp Pediatr [Internet]. 2020 [citado 2020 May 27];hpeds.2020-000356. Disponible en: https://doi.org/10.1542/ hpeds.2020-000356. [Epub ahead of print]

31. A Vanegas Ramirez 1, D Efe 1, M Fischer 1. Drug-induced vasculitis in a patient with COVID-19. J Eur Acad Dermatol Venereol [Internet]. 2020 [citado 2020 May 12]. Disponible en: https://doi.org/10.1111/jdv.16588. [Epub ahead of print]

32. Grandolfo, M., Romita, P., Bonamonte, D., Cazzato, G., Hansel, K., Stingeni, L., et al. Drug reaction with eosinophilia and systemic symptoms syndrome to hydroxychloroquine, an old drug in the spotlight. Dermatologic Therapy [Internet]. 2020 [citado 2020 May 12]. Disponible en: https://doi.org/10.1111/dth.13499. [Epub ahead of print]

33. Schwartz RA1, Janniger CK1. Generalized pustular figurate erythema A newly delineated severe cutaneous drug reaction linked with hydroxychloroquine. Dermatol Ther [Internet]. 2020 [citado 2020 May 12]. Disponible en: https://doi. org/10.1111/dth.13380. [Epub ahead of print] 